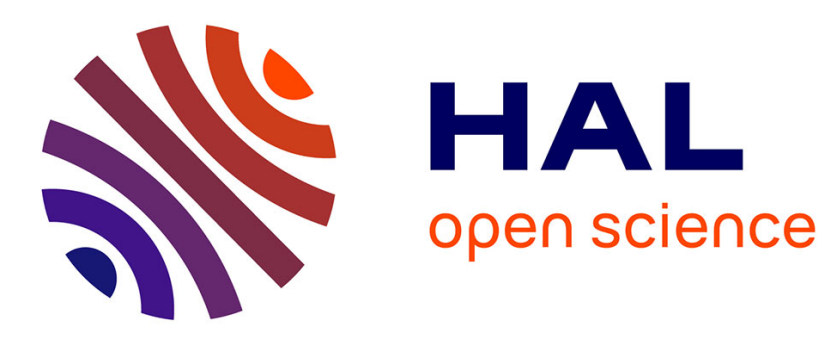

\title{
Linearization of radio frequency amplifiers using nonlinear Internal Model Control method
}

\author{
Smail Bachir, Claude Duvanaud
}

\section{To cite this version:}

Smail Bachir, Claude Duvanaud. Linearization of radio frequency amplifiers using nonlinear Internal Model Control method. AEÜ - International Journal of Electronics and Communications / Archiv für Elektronik und Übertragungstechnik, 2011, 65 (6), pp.495-501. 10.1016/j.aeue.2010.07.004 . hal00782014

\section{HAL Id: hal-00782014 \\ https://hal.science/hal-00782014}

Submitted on 29 Jan 2013

HAL is a multi-disciplinary open access archive for the deposit and dissemination of scientific research documents, whether they are published or not. The documents may come from teaching and research institutions in France or abroad, or from public or private research centers.
L'archive ouverte pluridisciplinaire HAL, est destinée au dépôt et à la diffusion de documents scientifiques de niveau recherche, publiés ou non, émanant des établissements d'enseignement et de recherche français ou étrangers, des laboratoires publics ou privés. 


\title{
Linearization of Radio Frequency Amplifiers Using NonLinear Internal Model Control method
}

\author{
S. Bachir*, C. Duvanaud \\ University of Poitiers, XLIM Laboratory, Department of SIC \\ Bât. SP2MI, Téléport 2, Bvd Marie et Pierre Curie, 86962 Futuroscope Chasseneuil Cedex, France \\ URL: http://www.sic.sp2mi.univ-poitiers.fr/ \\ Corresponding author: smail.bachir@univ-poitiers.fr
}

\begin{abstract}
In the present paper, the nonlinear Internal Model Control (IMC) method is introduced and applied to linearize high frequency Power Amplifiers (PAs). The IMC is based on the description of a process model and of a controller. It is shown that baseband frequency descriptions are suitable for the model and the controller. Their description parameters are derived from input and output modulation signals processed in Cartesian form. Simulation results are given to illustrate the design procedure and to demonstrate the performances of the IMC linearizer.
\end{abstract}

Key words: Power amplifiers, linearization technique, Internal Model Control, parameters identification, baseband signals, predistortion.

\section{Introduction}

Nonlinear system linearization of microwave components and radio-frequency circuits becomes a challenge and potential useful problem in the radiocommunication system research areas. Interest for Power Amplifier (PA) control is motivated by the increasing growth of the wireless communication systems which has lead to use digital modulation techniques such as (BPSK, QPSK, QAM, ...) with nonconstant envelope to improve spectral efficiency. As a result of the variable envelope modulation schemes, the improvement of the linearity of the PA becomes an objective of first importance for mobile communication systems. This is due to the nonlinear distortions and dynamical effects which generate unwanted spectrum components for the transmitted signal and lead to Adjacent Channel Power Ratio (ACPR) requirements.

Traditionally, techniques used for PAs linearization are based on feedback [1][2][3], feedforward [4][5], and predistortion [1][6][7]. Other structures guided to an efficient amplification exist like LInear amplification with

\footnotetext{
${ }^{*}$ Corresponding author

Email addresses: smail.bachir@univ-poitiers.fr (S. Bachir), claude.duvanaud@univ-poitiers.fr (C. Duvanaud) Bachir)$$
\text { URL: http://www.sic.sp2mi.univ-poitiers.fr/ (S }
$$

Preprint submitted to Elsevier
}

Nonlinear Components (LINC)[8], Combined AnalogLocked Loop Universal Modulator (CALLUM)[9], and Envelope Elimination and Restoration (EER)[10]. The predistortion technique involves the creation of an inverse characteristic complementary to the PA nonlinearity [11][12][13]. Performances can be improved by adding a feedback control to deal with external perturbations, parameter variations or operating frequency modifications. Design of conventional feedback requires considering carefully performances in term of gain-bandwidth product and stability [14][15][16]. The nonlinear IMC is an alternative solution using a model of the nonlinear system to be controlled [17]. If the model is a perfect representation of the nonlinear system, the controller can be design without concern for system stability. Furthermore, the control method of the IMC is very simple and comprehensive, leading to an easy tuning of the controller parameters.

For radio communications applications, the model and the controller are difficult to design because of the high operating frequency used and time delay introduced [18][19]. A solution consists in using baseband signals to describe the behavior of system parts. For feedback implementation, an error signal can be given by the comparison of the input and output envelope signals [20][21]. More advanced techniques used polar or 
cartesian signal formats to take into account the phase distortion [1][22][23]. In this paper, we proposed to design an IMC structure using Cartesian signal. The model and the controller are then described by mathematical expressions derived from measurements of the downconverted output signals and of the input modulating signal. Then, the technique results in the linearization of the overall microwave-transmitter.

Different stages leading to the description of the IMC structure are introduced and illustrated by nonlinear simulation results. At first, a model is established to describe the behavior of the transmitter and secondly a design of the nonlinear controller is presented. IMC is analyzed and compared to standard predistortion method trough simulation using $A D S$ and MATLAB softwares.

The remainder of this paper is organized as follows. In Section 2, the IMC method, based on continuoustime signals, is revised and applied to the PA linearization problem. Section 3 is devoted to the nonlinearity and frequency model description. In section 4 , we present simulation results with the linearizer structure, evaluating its performances from the two-tone test and from the transmission of a QPSK, 16-QAM and 64QAM digitally modulated signal. And, finally, Section 5 summarizes and concludes this paper.

\section{Internal Model Control}

IMC was originally developed for chemical engineering applications [24], and is considered as a robust control method. The IMC structure is shown in Fig. 1.

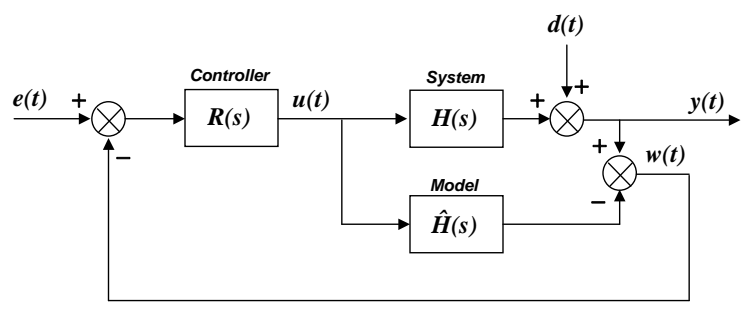

Figure 1: Schematic of the IMC structure

The structure uses an internal model $\hat{H}(s)$ in parallel with the system to be linearized $H(s)$. The control loop is augmented by a block filter $R(s)$, so-called the $I M C$ controller. In this structure, $s$ denotes a Laplace operator for continuous time-domain consideration.

\subsection{IMC analysis}

The closed loop transfer function describing the input $e(t)$ and the disturbance $d(t)$ to output $y(t)$ relationship is given by:

$$
\begin{aligned}
Y(s)= & \frac{R(s) H(s)}{1+R(s)(H(s)-\hat{H}(s))} E(s) \\
& +\frac{1-R(s) \hat{H}(s)}{1+R(s)(H(s)-\hat{H}(s))} D(s)
\end{aligned}
$$

The IMC loop computes the difference $w(t)$ between the system output $H(s)$ and the model output $\hat{H}(s)$. This residual signal $w(t)$ represents the effects of the disturbance $d(t)$ and of the modeling error between the model and the system. The IMC structure has advantages over conventional feedback control loop. If a perfect model is used, i.e. $\hat{H}(s)=H(s)$ there exists no feedback, and the closed loop system is affected only by the disturbance $d(t)$. In this case, the closed loop system has the transfer function:

$$
Y(s)=R(s) H(s) E(s)+(1-R(s) \hat{H}(s)) D(s)
$$

Noted that without disturbance the system is effectively open-loop hence no stability problems can arise. Also, if the system $H(s)$ is stable, which is the case for application to power amplifiers, the closed-loop will be stable for any stable controller $R(s)$. Furthermore, the controller can be designed as a feedforward controller in the IMC scheme.

An ideal control system, would suppress all disturbances and insure the instantly tracking of the input $e(t)$. From equation (2), the ideal control system is achieved for:

$$
R(s) . \hat{H}(s)=1
$$

So, the ideal choice for the controller $R(s)$ is the model inverse $\hat{H}(s)^{-1}$. For the investigated application, the IMC must allow to compensate the static non linearity of the amplifier. The model of the system includes a model of the nonlinear static characteristic and the IMC controller includes the inverse static characteristic. If the inversion of the static characteristic model is perfect, the IMC scheme allows a rejection of the disturbance and of the non linearity effects.

\subsection{Design procedure for PA linearization}

The block diagram of IMC is shown in Fig. 2. All signal designations refer either to complex baseband signals and don't depend on the modulation format.

The proposed IMC structure allows linearizing the transmitter composed of the I/Q modulator, amplifier and demodulator blocks. The output of the demodulator is compared with the output of the baseband model of 


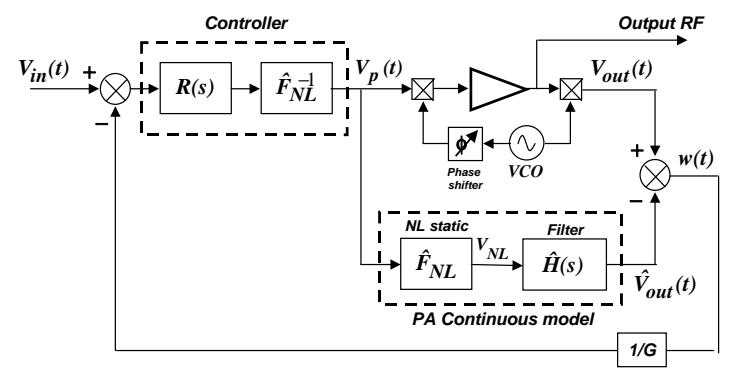

Figure 2: Baseband equivalent scheme of the IMC structure

the amplifier and the resulting error signal $w(t)$, normalized according to PA gain noted $G$, is used to modify the input modulation signal.

The critical point in the IMC structure is the description of the inverse amplifier static nonlinearity. A solution consists in using a complex polynomial function, composed by even terms, to describe the inverse AM/AM and AM/PM characteristics. The main advantage of such models is that they are linear-in-parameters allowing Least Mean Square (LMS) estimation techniques.

\section{Baseband PA characterization}

In IMC structure (Fig. 2), it is necessary to have a description of the process in continuous time domain. In this paper, a special case of Volterra series, the Hammerstein memory polynomial, is used.

\subsection{PA model description}

The nonlinear block presented here operates on baseband quadrature I/Q time-domain waveforms [17]. The complex low-pass equivalent representation of the communication signal is used to avoid the high sampling rate required at the carrier frequency.

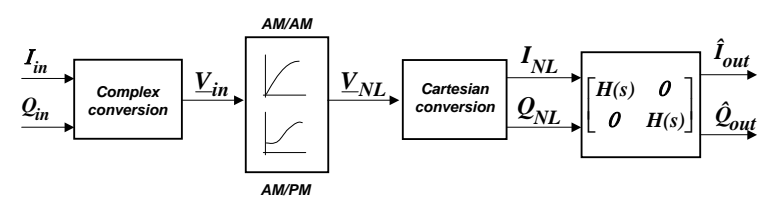

Figure 3: Radio frequency power amplifier model

As shown in Fig. 3, the two-box model includes a memoryless nonlinearity and a filter matrix. The complex gain gives a nonlinear version $\underline{V}_{N L}=I_{N L}+j \cdot Q_{N L}$ of the transmitted input signal $\underline{V}_{i n}=I_{i n}+j \cdot Q_{i n}$ according to the polynomial function composed by even terms which produces harmonic distortions inside the PA bandwidth:

$$
\underline{V}_{N L}=\sum_{k=0}^{P} \underline{c}_{2 k+1} \cdot\left|\underline{V}_{i n}\right|^{2 k} \cdot \underline{V}_{i n}
$$

where $\underline{c}_{2 k+1}$ are the complex power series coefficients.

The dynamical model including memory effects caused by the PA may be expressed with a differential equation. As shown in Fig. 3, the input $\underline{V}_{N L}$ to output $\hat{\underline{V}}_{\text {out }}=\hat{I}_{\text {out }}+j \hat{Q}_{\text {out }}$ relation of this $n^{\text {th }}$ order filter can be written as:

$$
H(s)=\frac{\hat{I}_{\text {out }}}{I_{N L}}=\frac{\hat{Q}_{\text {out }}}{Q_{N L}}=\frac{\sum_{k=0}^{m} b_{k} \cdot s^{k}}{s^{n}+\sum_{k=0}^{n-1} a_{k} s^{k}}
$$

where the coefficients $\left\{a_{k}\right\}$ and $\left\{b_{k}\right\}$ are real scalars that define the model.

\subsection{Identification algorithm}

The parameters $a_{k}, b_{k}$ and $\underline{c}_{k}$ of previous PA model have been identified by Output-Error technique (see also [25]). Thus, we define the transposed parameter vector:

$$
\underline{\theta}=\left[\begin{array}{lllllll}
a_{0} \cdots a_{n-1} & b_{0} & \cdots & b_{m} & \underline{c}_{1} & \cdots & \underline{c}_{2 P+1}
\end{array}\right]^{T}
$$

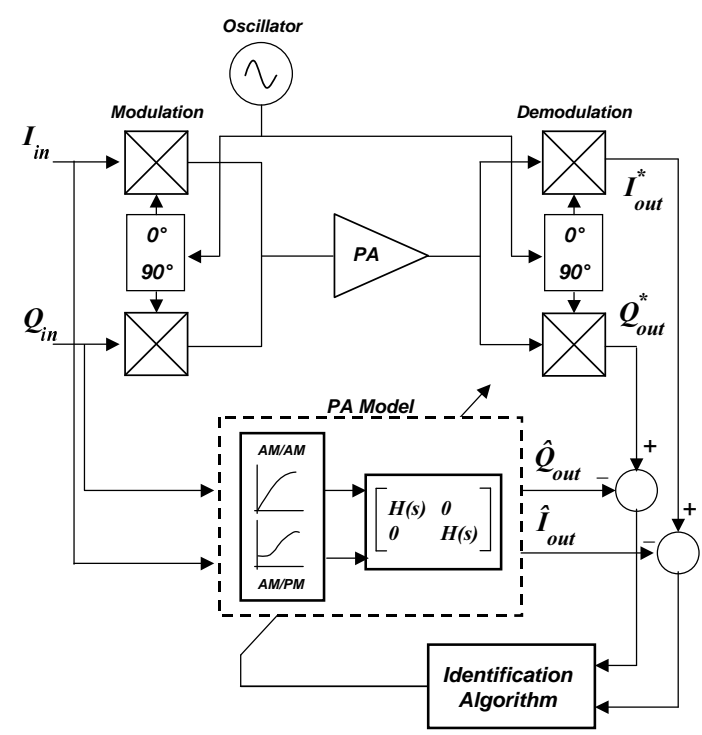

Figure 4: PA identification scheme

With $K$ values of baseband input vector $\left(I_{i n_{k}}, Q_{i n_{k}}\right)$ and output vector $\left(I_{\text {out }_{k}}^{*}, Q_{\text {out }_{k}}^{*}\right)$, off-line parameter esti- 
mation is based on minimization of a quadratic multivariable criterion defined as:

$$
\begin{aligned}
J & =\sum_{k=1}^{K}\left(I_{\text {out }_{k}}^{*}-\hat{I}_{\text {out }_{k}}\right)^{2}+\left(Q_{\text {out }_{k}}^{*}-\hat{Q}_{\text {out }_{k}}\right)^{2} \\
& =\sum_{k=1}^{K}\left(\varepsilon_{I_{k}}^{2}+\varepsilon_{Q_{k}}^{2}\right)
\end{aligned}
$$

As shown in Fig.4, we obtain the optimal values of $\underline{\theta}$ by Non Linear Programming techniques. Practically, Marquardt's algorithm [26] is used for the the minimization of quadratic criterion based on the error between measured data and estimation such as:

$$
\hat{\theta}_{i+1}=\underline{\hat{\theta}}_{i}-\left\{\left[J_{\theta \theta}^{\prime \prime}+\lambda \cdot I\right]^{-1} \cdot J_{\theta}^{\prime \prime}\right\}_{\hat{\theta}=\underline{\theta}_{i}}
$$

$J_{\theta}^{\prime}$ and $J_{\theta \theta}^{\prime \prime}$ are respectively gradient and hessian such as:

$J_{\theta}^{\prime}=-2 \sum_{k=1}^{K}\left(\underline{\varepsilon}_{I_{k}}^{T} \cdot \underline{\sigma}_{I_{k, \underline{\theta}}}+\underline{\varepsilon}_{Q_{k}}^{T} \cdot \underline{\sigma}_{Q_{k, \underline{\theta}}}\right)$

$J_{\theta \theta}^{\prime \prime} \approx 2 \sum_{k=1}^{K}\left(\underline{\sigma}_{I_{k, \underline{g}}} \cdot \underline{\sigma}_{I_{k, \underline{\theta}}}^{T}+\underline{\sigma}_{Q_{k, \underline{\theta}}} \cdot \underline{\sigma}_{Q_{k, \underline{\theta}}}^{T}\right)$

$\lambda$ is the monitoring parameter,

The sensitivity $I Q$ functions $\underline{\sigma}_{I_{k, \underline{\theta}}}=\frac{\partial \hat{\underline{I}}_{\text {out }}}{\partial \underline{\underline{\theta}}}$ and $\underline{\sigma}_{Q_{k, \theta}}=\frac{\partial \hat{\underline{Q}}_{o u t}}{\partial \underline{\underline{\theta}}}$ are obtained, for each parameter, by partial differentiation of global PA model (Eqs. 4-5).

Identification results are shown for a MESFET power amplifier, used at a center frequency of $900 \mathrm{MHz}$. The input signal is a pseudo random binary sequences (PRBS) at rate of $60 \mathrm{Mb} / \mathrm{s}$, filtered by a low pass rootraised cosine with $\alpha=0.35$. Optimization algorithm and all data processing are performed using Matlab Mathworks. For the amplitude and phase identification, fifth order polynomial expressions and $1^{\text {st }}$ order filter $H(s)$ are used and allow achieving a good agreement between the amplifier behavior and its estimation such as:

$$
\left\{\begin{array}{l}
\underline{V}_{N L}=\left(\underline{c}_{1}+\underline{c}_{3} \cdot\left|\underline{V}_{i n}\right|^{2}+\underline{c}_{5} \cdot\left|\underline{V}_{i n}\right|^{4}\right) \cdot \underline{V}_{\text {in }} \\
\underline{V}_{\text {out }}(s)=H(s) \cdot \underline{V}_{N L}(s)=\frac{a_{0}}{s+a_{0}} \cdot \underline{V}_{N L}(s)
\end{array}\right.
$$

After 8 iterations, we obtain the following parameters:

$$
\underline{\hat{\theta}}=\left[\begin{array}{l}
c_{1}=6,6537-j 1,61356 \\
c_{3}=-2,3588+j 9,0722.10^{-1} \\
c_{5}=4,51456.10^{-1}-j 1,7331.10^{-1} \\
a_{0}=4,7493 \cdot 10^{8}
\end{array}\right]
$$
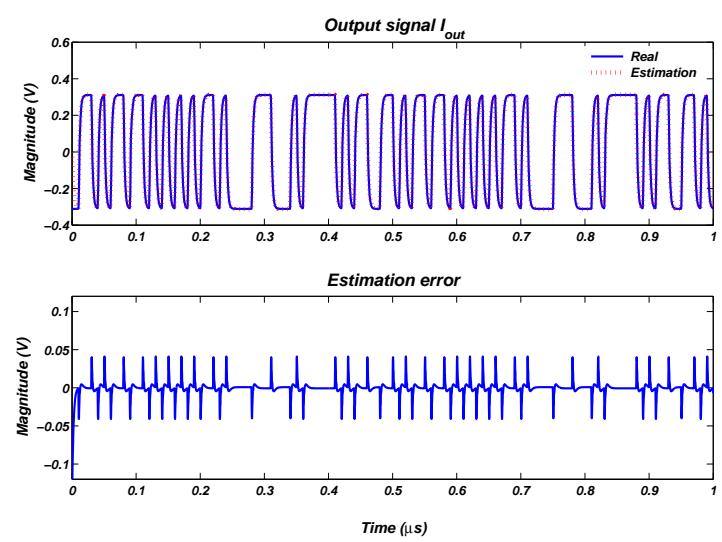

Figure 5: Comparison of time-domain measurement and estimation

Figure (5) allows a comparison between measured output waveform and estimation on $I$ channel. As can be seen, even if the amplifier is driven near saturation, the non linear behavioral of the amplifier is successfully described by the obtained model.

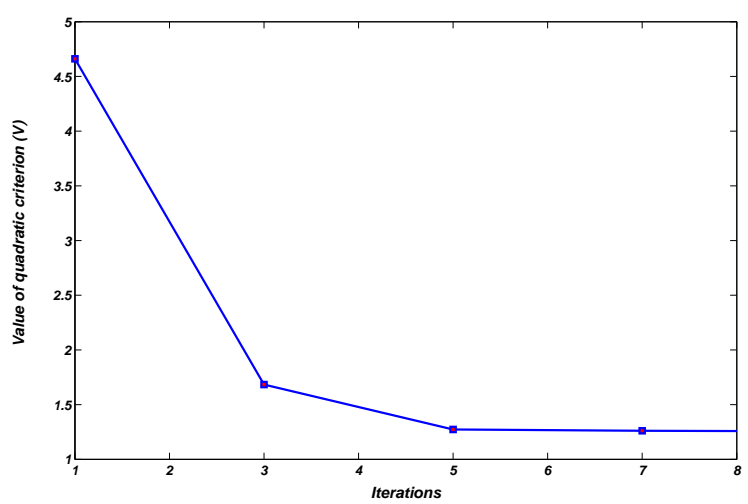

Figure 6: Convergence of estimation error during identification

The estimation error evaluated by a quadratic criterion value $J$ (Eq. 7) is an indication of the correspondance degres of the obtained model and the actual PA. Figure (6) shows the convergence of this coefficient to optimal value corresponding to the output noise and modeling errors [25].

The dynamic characteristic of the PA system can be described by a MIMO coupled filter. The real filter $H(s)$ and the obtained filter $\hat{H}(s)$ characteristics are represented in Fig. (7) by the gain and phase curves. Note that the cut off frequency of the corresponding filter is around $76 \mathrm{MHz}$. 

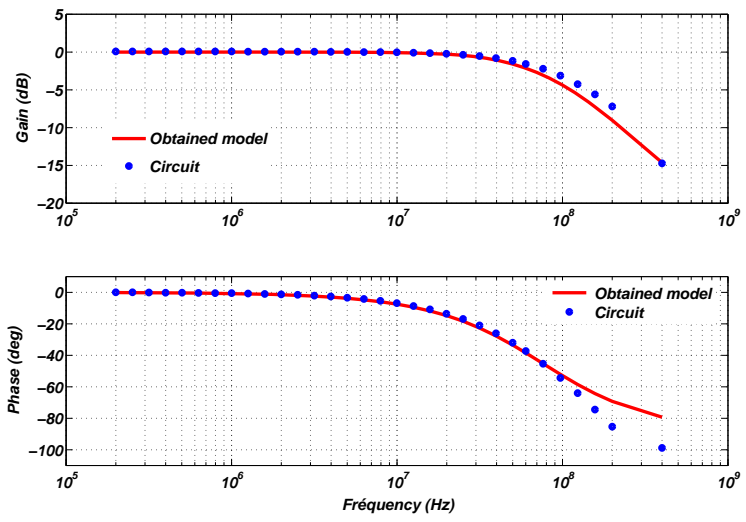

Figure 7: Comparison between a frequency responses of the PA circuit and the obtained filter

\section{Simulation and investigation}

The IMC structure is evaluated by nonlinear simulations using circuit envelope algorithm with Agilent ADS simulator. The described class AB PA presented in figure (8), is a single stage structure composed of a MESFET device by Infineon (CLY 5). The behavior of the transistor is described by a table based model of the nonlinear drain current source associated to a junction model for the gate to source capacitance.

The matching topology is designed to ensure optimum power and efficiency performances at the $900 \mathrm{MHz}$ operating frequency. The output matching has a low pass $\mathrm{T}$ structure composed of a capacitor to ground and two transmission lines.

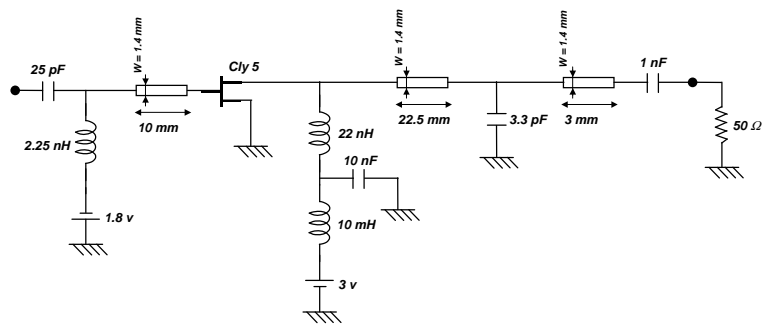

Figure 8: PA circuit implemented on ADS software

Figures (9) and (10) shows the AM-AM and AM-PM conversion characteristics for the studied amplifier. The $1 \mathrm{~dB}$ compression point is achieved for an input power of $2.4 \mathrm{dBm}$. The amplifier behaviour has been simulated using the Harmonic Balance algorithm and a sinusoidal signal at $900 \mathrm{MHz}$.

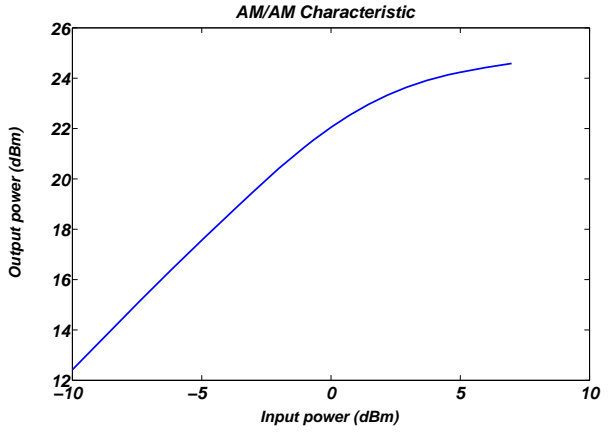

Figure 9: AM/AM conversion characteristic

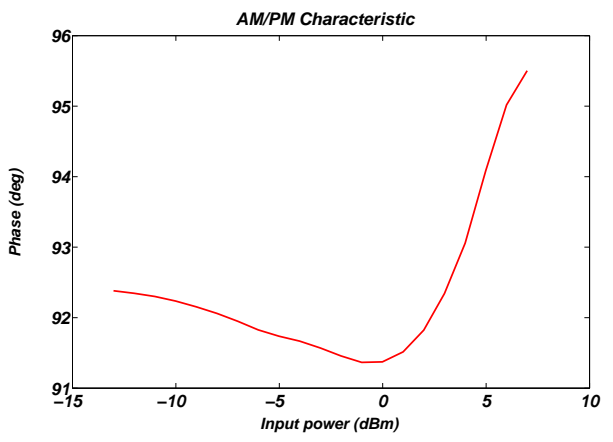

Figure 10: AM/PM conversion characteristics

\subsection{Inverse PA characteristic model}

In linearizer architecture illustrated in figure (2), an inverse characteristic $\hat{F}_{N L}^{-1}$ is included to compensate the PA static nonlinearities. This memoryless function take into account simultaneous gain and phase characteristics and can be modeled with a complex polynomial series [6][11]. Contrary to $F_{N L}$, the inverse function $F_{N L}^{-1}$ describe the relationship between complex envelope $\underline{V}_{i n}$ and $\underline{V}_{\text {out }}$ such as:

$$
\begin{aligned}
\underline{V}_{\text {in }}(k) & =F_{N L}^{-1}\left(\underline{V}_{\text {out }}(k)\right) \\
& =\sum_{q=0}^{Q} d_{2 q+1}\left|\underline{V}_{\text {out }}(k)\right|^{2 q} \underline{V}_{\text {out }}(k)
\end{aligned}
$$

The ultimate step consists in searching approximation of the complex parameters $\underline{d}_{2 k+1}$ using the envelope magnitude and phase distortions. Thus, the AM/AM and AM/PM characteristics are used to optimize a polynomial function by Least Mean Square (LMS) algorithm [27]. A solution for the coefficients is obtained by minimizing the mean-squared error between the measured and the modeled output under low frequency signal such as:

$$
\underline{\hat{\theta}}_{d}=\left(\phi^{H} \phi\right)^{-1} \phi^{H} \underline{V}_{i n}
$$


where :

(.) ${ }^{H}$ denotes transpose-conjugate transformation

$\underline{\hat{\theta}}_{d}=\left[\begin{array}{llll}\underline{d}_{1} & \underline{d}_{3} & \cdots & \underline{d}_{2 Q+1}\end{array}\right]^{T}$ is the vector of polynomial parameters,

$\underline{V}_{\text {in }}$ and $\underline{V}_{o u t}$ are the measured input and output complex envelopes,

$\phi=\left[\begin{array}{llll}\underline{\varphi}_{1} & \underline{\varphi}_{2} & \cdots & \underline{\varphi}_{K}\end{array}\right]$ is the regression matrix,

$\underline{\varphi}_{k}=\left[\begin{array}{llll}V_{\text {out }_{k}} & V_{\text {out }_{k}}\left|V_{\text {out }_{k}}\right|^{2} & \cdots & V_{\text {out }_{k}}\left|V_{\text {out }_{k}}\right|^{2 Q}\end{array}\right]^{T}$ is the regression vector,

and $\underline{V}_{\text {out }_{k}}$ is a $k^{\text {th }}$ sampled input.

Noted that for these estimations, the regression vector $\underline{\varphi}_{k}$ is not correlated with the measured input $\underline{V}_{i n}$.
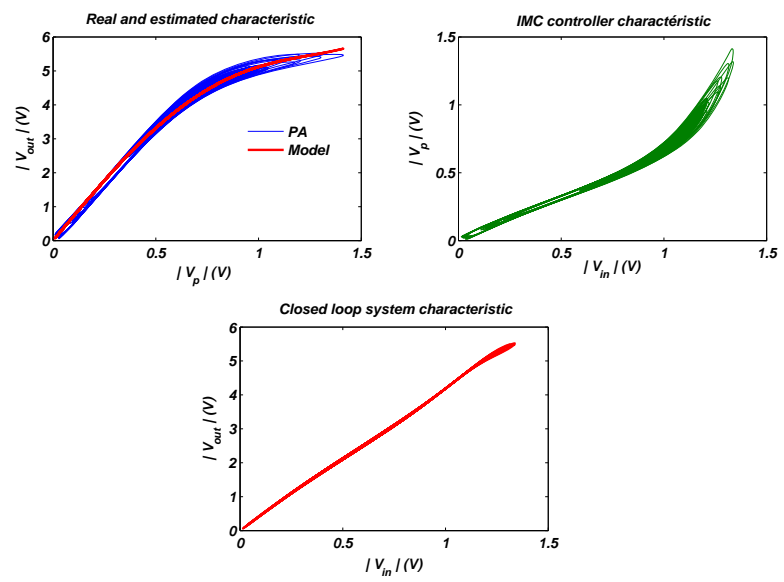

Figure 11: Comparison of the dynamic envelope variations at the output of amplifier, model, controller ans the dynamic AM/AM characteristic of the IMC system.

In practice, the PA characteristics is performed by a sinusoidal excitation applied on baseband inputs $I_{\text {in }}$ and $Q_{i n}$ at fixed low frequency and high input level. In these conditions, the PA filtering effects are assumed negligible according to non linear dynamics. The input-output curves are obtained by measuring the output gain and phase as a function of input level.

For illustration, on figure (11) are presented the dynamic envelope variations of amplifier and model output for a $16-\mathrm{QAM}$ at a bit rate of $2.5 \mathrm{Mb} / \mathrm{s}$, modulated at $900 \mathrm{MHz}$ and shaped with a raised-cosine pulse with $35 \%$. This signal is applied to the IMC system. Also plotted are the dynamic enveloppe at the output of controller and of overall IMC system versus the instantaneous input enveloppe. This last curve allows to verify that the nonlinear behavior and the hysteresis effects are reduced, showing the interest of the IMC system.

\subsection{Linearization performances}

To evaluate the effectiveness of the proposed linearization technique in suppressing spectral regrowth, we compare the power spectral density of the PA output without linearizer, with memoryless predistortion and with IMC memory controller.

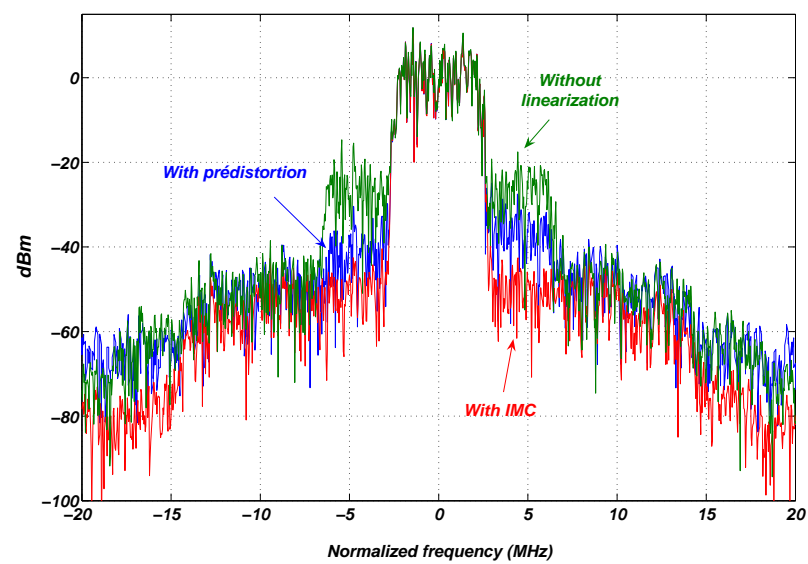

Figure 12: Output spectrum for the amplifier without linearization, with predistorsion and with IMC.

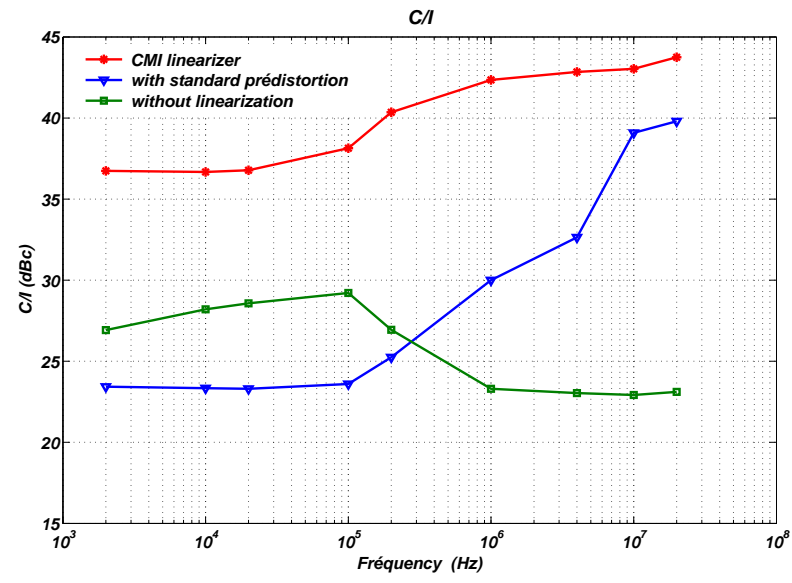

Figure 13: Carrier to intermodulation ratio versus carrier frequency difference

Here, the baseband input is 4-QPSK digitally modulated signal at a bit rate of $5 \mathrm{Mb} / \mathrm{s}$. Figure 12 shows the output spectrum for an output power backoff of 3 
dB. Using IMC structure, an improvement of approximatively $10 \mathrm{~dB}$ is obtained according to standard predistortion and of $30 \mathrm{~dB}$ according to PA without linearization.

Due to memory effects, linearization performances can depend on baseband signal frequency. To verify the performances of IMC, simulations have been performed for a two tones excitation with a difference of $2 \mathrm{KHz}$ to $20 \mathrm{MHz}$ between the carrier frequencies. Results are plotted on figure (13) in terms of carrier to third order intermodualtion ratio $\mathrm{C} / \mathrm{I}$ in $\mathrm{dBc}$. For the memoryless predistortion designed with the previous $2.5 \mathrm{Mb} / \mathrm{s}$ 16-QAM modulation, performances are reduced and degraded at low baseband frequencies. Whereas, the IMC system allows to improve the nonlinear performances for the whole bandwidth considered.

If there is a signal with non-constant envelope at the PA's input, each of its samples will be amplified with different gain and the introduced phase shift will differ according to the input signal amplitude. As a consequence, the PA output signal will suffer from nonnegligible nonlinear distortion. Thus, for example in the case of 16-QAM and 64-QAM modulations shown in figures (14) and (15), the constellation point near the saturation will be more deformed in the case of PA without linearizer. These figures shows too the amelioration of impairments of this nonlinearity in constellation diagrams in the case of IMC feedback controller.

\section{Conclusion}

In this paper, nonlinear internal model control was introduced and applied to power amplifier linearization with different baseband signals. Design of the IMC system, using baseband signal, is described. Simulations are presented to demonstrate the interest of the technique. For a $16-\mathrm{QAM}$ at a bite rate of $2.5 \mathrm{Mb} / \mathrm{s}$, IMC allows to reduce nonlinear and hysteresis effects on the dynamic envelope characteristic. Simulated performances show improvement of the ACPR of $30 \mathrm{~dB}$ for the amplifier at high output power, and $10 \mathrm{~dB}$ in comparison to predistortion. Furthermore, IMC allows to improve performances in term of linearity for different output levels and signal bandwidths. Compared with classical Cartesian feedback and feedforward structures, the robustness and stability of the proposed linearizer is improved.

Our next objective is to implement the proposed linearizer architecture for an industrial amplifier in cellular phones or RF base-station. In this case, we can study the robustness improvement with regard to carrier phase shifts in the linearizer branches or stability according to on-line devices variation.

\section{References}

[1] P.B. Kenington, High-Linearity RF Amplifier Design, Artech House, London 2000.

[2] S.C. Cripps, Advanced techniques in RF power amplifier design, Artech House, Boston, 2002.

[3] F.H. Raab, P. Asbeck, S.C. Cripps, P.B. Kenington, Z.B. Popovic, N. Pothecary, J.F. Sevic, and N.O. Sokal, Power amplifiers and transmitters for $R F$ and microwave, IEEE Trans. Microwave Theory. and Tech. VOL. 50, pp. 814-826, 2002,

[4] N. Pothecary, Feedforward Linear Power Amplifiers, Norwood, MA: Artech House, 1999.

[5] P.B. Kenington, P.A. Warr, and R.J. Wilkinson, Analysis of instability in feedforward loop, Electronics Letters, VOL. 33, pp. 16691671, September 1997.

[6] Y. Nagata, Linear amplification technique for digital mobile communications, 39 ${ }^{\text {th }}$ Proc. IEEE Vehicular Tech. Conf. pp. 159164, San Francisco 1989.

[7] J.S. Kenney, W. Woo, L. Ding, R. Raich, H. Ku and G.T. Zhou, The impact of Memory Effects on Predistortion Linearization of $R F$ Power Amplifiers, Proc. of $8^{\text {th }}$ Int. Sym. on Microwave and Optical Technology ISMOT'2001, pp. 189-193, Montreal, Canada, June 2001.

[8] D.C. Cox, Linear amplification with nonlinear components, IEEE Transactions on Communications, VOL. COM-22, pp. 1942-1945, 1974

[9] D.J. Jennings and J.P. McGeEHan, A high-efficiency RF transmitter using VCO-derived synthesis : CALLUM, IEEE Transactions on Microwave Theory and Techniques, VOL. 47, NO. 6, pp. 715721, June 1999.

[10] D. Su and W. McFarland, An IC for linearizing RF power amplifiers using envelope elimination and restoration, in ISSCC Digest of Technical Papers, pp. 54-55, 1998.

[11] J.K. Cavers, Amplifier linearization using a digital predistorter with fast adaptation and low memory requirements, IEEE Trans. Vehicular Technology, VOL. 39, NO. 04, pp. 374-382, Nov. 1990.

[12] M. Johansson and T. Mattson, Linearised high-efficiency power amplifier for PCN, Electronics Letters, VOL. 27, NO. 9, pp. 762764, Avril 1991

[13] J. Kim and K. Konstantinou, Digital predistortion of wideband signals based on power amplifier model with memory, Electronics Letters, VOL. 37, pp. 1417-1418, 2001.

[14] A.A.M. Saleh and J. Salz, Adaptive linearization of power amplifiers in digital radio systems, Bell System Tech. J. VOL. 62, NO. 4, pp. 1019-1033, April 1983.

[15] A.V. Malipatil and Y.-F. Huang, Amplifier predistortion using unscented Kalman filtering, IEEE $6^{\text {th }}$ Workshop on Signal Processing Advances in Wireless Communications, pp. 221-225, June 2005.

[16] M. ODroma, E. Bertran, J. Portilla, N. Mgebrishvili, S. Donati Guerrieri, G. Montoro, T.J. Brazil and G. Magerl, On Linearisation of Microwave-Transmitter Solid-State Power Amplifiers, International journal of RF and microwave computer-aided engineering, VOL. 15, NO. 5, pp. 491-505, 2005.

[17] M. Djamai, Contribution a la modlisation et la linarisation par prdistorsion numrique adaptative en bande de base des amlificateurs de radiocommunication, $\mathrm{Ph}$. D. Thesis, University of Poitiers, France, 2008.

[18] H. Ku, M.D. McKinley and J.S. Kenney, Quantifying memory effects in RF power amplifiers, IEEE Transactions on Microwave 

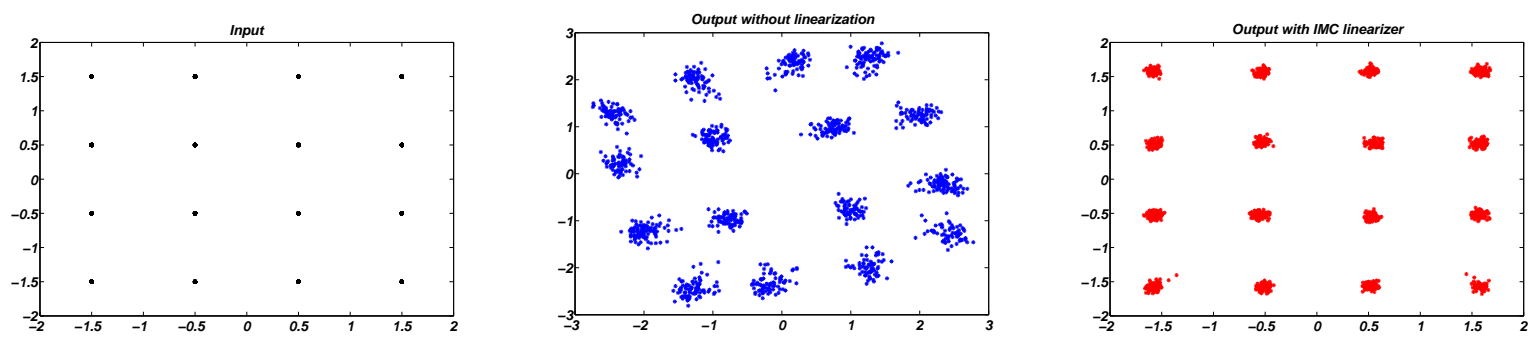

Figure 14: Constellation diagrams for 16-QAM signal
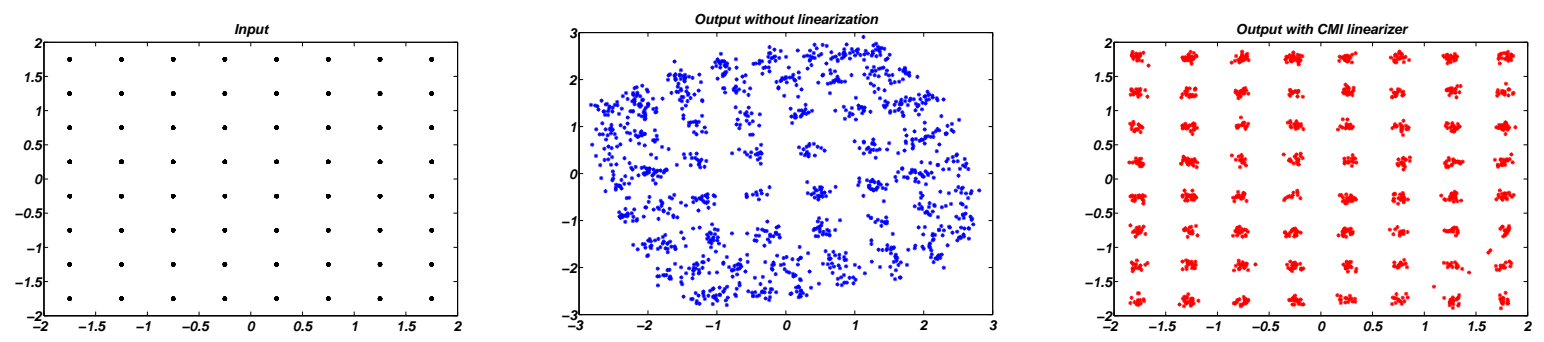

Figure 15: Constellation diagrams for 64-QAM signal

Theory and Techniques, VOL. 50, NO. 12, pp. 2843 - 2849, December 2002.

[19] A.E. Nordsjö, An algorithm for adaptive predistortion of certain time-varying nonlinear high-power amplifiers, RADAR 2002, pp. 469-473, 15-17 Oct 2002.

[20] T. Elgeryd, Iterative algorithms for linearising non-linear systems by digital predistortion, Master thesis report, Royal Institute of Technology, Stockholm 2002.

[21] R. Marsalek, P. Jardin, G. Baudoin, From post-distortion to predistortion for power amplifiers linearization, IEEE Communications Letters, VOL. 07, pp. 308-310, July 2003.

[22] S.C. Cripps, RF Power Amplifiers for Wireless Communication, Norwood, MA: Artech House, 1999.

[23] A. Katz, Linearization : Reducing Distortion in Power Amplifiers, IEEE microwave magazine, pp. 37-39, December 2001.

[24] C. Garcia and M. Morari, Internal model control. A unifiying review and some new results, Ind. Eng. Chem. Process Des. Dev., VOL. 21, pp. 472-484, 1982.

[25] M. Djamai, S. Bachir and C. Duvanaud, Modeling and identification of continuous-time system for RF amplfiers, European Microwave Conference, Amsterdam, Holland, 2008.

[26] D. W. Marquardt, An Algorithm for least-squares estimation of non-linear parameters, Soc. Indust. Appl. Math, VOL. 11, NO. 2, pp. 431-441, 1963.

[27] L. Ljung, System identification: Theory for the user, $2^{\text {nd }}$ edition, Prentice Hall, USA, 1999. 